\title{
GALACTIC KINEMATICS ON THE BASIS OF MODERN PROPER MOTION DATA
}

\author{
M. MIYAMOTO \\ National Astronomical Observatory \\ Mitaka, Tokyo 181, Japan
}

\begin{abstract}
An accumulation of high precision astrometric data in conjunction with high-precision monitoring of the Earth's orientation, motivates "Galactic Astronomy". As regards local kinematics, all of the three components of both the vorticity and the shear of stars can be completely determined, in addition to the velocity ellipsoid. We can now be released from the constraint of the "axisymmetric" galaxy. The determination of the proper motion of the LMC will be crucial to understanding the global structure and dynamics of the Galaxy with the dark halo and MACHO's motions.
\end{abstract}

\section{Introduction}

With an accumulation of accurate astrometric data for a huge number of stars in a large galactic volume, provided by astrographs (NPM and SPM projects), photoelectric meridian circles equipped with the 2-D CCD micrometer (La Palma, Bordeaux, USNO, and Tokyo Meridian Circles), and HIPPARCOS, we are now entering the second brilliant era of the "Stellar Astronomy" (cf. Trumpler and Weaver 1953) since the time of Oort and Lindblad. During the first era, attention focused on probing Galactic Rotation and Stellar Populations, while in the current era we shall take a great interest in clarifying the Galactic Dark Halo.

Since the availability of proper motion data provides a primary impetus to Stellar Astronomy, the present review talk concentrates on the propermotion analysis based on a large astrometric catalogue (ACRS) available today. Two topics are discussed: the local kinematics of the Galaxy and the detection of the LMC proper motion. 


\section{Local Kinematic Parameters of the Galaxy}

The precessional constant plays a primary role in the reference frame for accurate proper motions. In order to obtain absolute proper motions, we need precise knowledge of the motion of the observationally accessible origin (the equator and equinox) of the reference frame, with reference to a background inertial frame. Planetary precession is determined to a sufficient accuracy (Lieske et al. 1977). A long-standing question is the accurate determination of the luni-solar precession.

In the first place, the change in the orientation of the observer's platform - the earth - should be monitored with reference to fixed points (QSO's) observable on the celestial sphere. Alternatively, the proper motions of stars determined directly with reference to background fixed points (galaxies) would be absolute, independent of the Earth's orientation. Such an idea was proposed in the 1930's by W.H.Wright, and is now applied successfully to the geodesic VLBI observations for monitoring the Earth's orientation, and to the NPM and SPM projects for the absolute determination of proper motions. However, we make the analysis of the proper motion data in rather classical fashion, for the moment, since the ideal observations mentioned above are not yet definitive. This situation will not change in the analysis of the proper motion data to be provided by the HIPPARCOS Catalogue.

Nowadays, we have the nominal luni-solar precessional constant to an accuracy of eight figures: $\psi=5038^{\prime \prime} .7784 /$ cy at J2000.0 (IAU (1976)). The present value of the constant results from Fricke's correction $\Delta p$ to Newcomb's value at the fourth and fifth digits (Fricke 1977). Fricke also determined the so-called fictitious equinoctial motion $\Delta e$, which is inherent in the optical determination of the equinox. The current fundamental reference system FK5 was constructed on the basis of Fricke's corrections (Fricke et al. 1988).

The importance for galactic astronomy of settling the fifth and sixth digits of the precessional constant should be stressed here. The fifth digit corresponds to the order of magnitude of $0^{\prime \prime} .1 / \mathrm{cy}(1 \mathrm{mas} / \mathrm{yr})$ and the galactic rotation indicated by the combination of the Oort constants $\mathrm{B}-\mathrm{A}$ is about $0^{\prime \prime} .5 / \mathrm{cy}(5 \mathrm{mas} / \mathrm{yr})$. On the other hand, the rotation of the galactic warp around the axis joining the sun and the galactic center is about $0^{\prime \prime} .05 / \mathrm{cy}$ ( $0.5 \mathrm{mas} / \mathrm{yr})$, as mentioned later. Therefore, at these digits the proper motion analyses for determining the precessional constant is strongly coupled to the general galactic rotation.

Now, in the galactic rectangular coordinate system $\left(x_{1}, x_{2}, x_{3}\right)$ with the origin at the sun and the three axes pointing to the galactic center, the direction of galactic rotation, and the north galactic pole, respectively, 
the three-dimensional Ogorodnikov-Milne model for the systematic stellar velocity field at the solar neighborhood $\boldsymbol{r}\left(=x_{1} \boldsymbol{i}+x_{2} \boldsymbol{j}+x_{3} \boldsymbol{k}\right)$ is expressed as

$$
\boldsymbol{V}=\boldsymbol{S}+\boldsymbol{\nabla} Q+\boldsymbol{\omega} \times \boldsymbol{r}
$$

where

$$
\begin{gathered}
Q=\frac{1}{2} \sum_{i, j} D_{i j}^{+} x_{i} x_{j}, \\
\boldsymbol{\omega}=D_{32}^{-} i+D_{13}^{-} j+D_{21}^{-} \boldsymbol{k}=\frac{1}{2} \operatorname{rot} \boldsymbol{V}, \\
D_{i j}^{ \pm}=\frac{1}{2}\left(\frac{\partial V_{i}}{\partial x_{j}} \pm \frac{\partial V_{j}}{\partial x_{i}}\right) \text { for } i, j=1,2,3,
\end{gathered}
$$

and $\boldsymbol{S}\left(S_{1}, S_{2}, S_{3}\right)$ is the mean flow (centroid) at the sun, so that the solar motion is given by $-\boldsymbol{S} . D_{i j}^{+}$denotes the shear of stars in the $\left(x_{i}, x_{j}\right)$-plane, $D_{i i}^{+}$the dilatation, and $D_{32}^{-}, D_{13}^{-}$, and $D_{21}^{-}$the rotations of stars around the $x_{1}, x_{2}$, and $x_{3}$ axes, respectively. All of these kinematic parameters are evaluated at the position of the sun. If the stellar velocity field is axisymmetric or $V_{R}=0, D_{12}^{+}$and $D_{21}^{-}$are reduced to the familiar Oort constants $A$ and $B$, respectively.

The equations of condition for least squares fitting are derived from eqs. (1)-(4). Given radial velocity data together with proper motion data, then we can solve these equations for all twelve unknowns $\left(S_{1}, S_{2}, S_{3}, \omega_{1}, \omega_{2}\right.$, $\left.\omega_{3}, D_{12}^{+}, D_{13}^{+}, D_{23}^{+}, D_{11}^{+}, D_{22}^{+}, D_{33}^{+}\right)$. It is noticed that nine parameters of the twelve - all except $D_{i i}^{+}$'s - can be completely determined on the basis of proper motion data only. We profit here from the two-dimensionality, the proper motions. The proper motions in stellar kinematics are twice as informative as radial velocities, if combined with a suitable distance estimate.

Hereafter, we concentrate on the determination of the nine parameters $S_{1}, \ldots, D_{23}^{+}$. The equations of condition are reduced to

$$
\left[\begin{array}{l}
\mu_{\alpha} \cos \delta \\
\mu_{\delta}
\end{array}\right]=\boldsymbol{M} \boldsymbol{X} \quad \text { with } \boldsymbol{X}^{T}=\left(S_{1} S_{2} S_{3} \omega_{1} \omega_{2} \omega_{3} D_{12}^{+} D_{13}^{+} D_{23}^{+}\right)
$$

where the $2 \times 9$ elements of the matrix $\boldsymbol{M}$ are given by Miyamoto and Sôma (1993) (referred to as Paper I), where in eq.(30), " $\alpha-\alpha_{\mathrm{GP}}$ " is substituted for " $\alpha$ ".

The rotation vector $\boldsymbol{\omega}$ deserves comment. If we have proper motions $\left(\mu_{\alpha}, \mu_{\delta}\right)$ given in a rigorously non-rotating reference frame, then, we can derive from eq.(5) a solution for the general galactic rotation $\boldsymbol{\omega}$ exclusively. However, the conventional reference frame, FK5, is still considered to be imprecise at the order of magnitude of $0^{\prime \prime} .1 / \mathrm{cy}(1 \mathrm{mas} / \mathrm{yr})$. Therefore, the 
proper motions $\left(\mu_{\alpha}, \mu_{\delta}\right)$ given in the FK5 system contain a contribution from the rotation of the frame itself. This situation will remain in the HIPPARCOS Catalogue to be released, since HIPPARCOS could not observe QSO's as fixed points on the celestial sphere.

Thus, the equatorial rectangular components of $\boldsymbol{\omega}$ in eq.(5) should be written as

$$
\left[\begin{array}{l}
\omega_{1} \\
\omega_{2} \\
\omega_{3}
\end{array}\right]=N\left[\begin{array}{l}
D_{32}^{-} \\
D_{13}^{-} \\
D_{21}^{-} \\
\Delta p \\
\Delta e+\Delta \lambda
\end{array}\right],
$$

where the $3 \times 5$ elements of the matrix $\boldsymbol{N}$ are trigonometric functions of the directions of the $x_{1}, x_{2}$, and $x_{3}$ axes and the ecliptic pole. $\Delta p, \Delta e$, and $\Delta \lambda$ denote the luni-solar precessional correction, the fictitious equinoctial motion correction, and the planetary precessional correction to the FK5 system, respectively.

It is noticed here that eq.(6) gives only three conditions for the five unknowns $D_{32}^{-}, D_{13}^{-}, D_{21}^{-}, \Delta p$, and $\Delta e+\Delta \lambda$. Therefore, applying least squares to eqs.(5)-(6), we can determine at most three designated unknowns of the five. This situation is a fundamental limitation on the analysis of proper motions. Thus, the important point to note is that the determination of the rotation left in the reference frame depends on a model of the galactic velocity field, while the determination of the galactic velocity field depends on the rotation left in the reference frame. In order to deal with this shortcoming, what we can do is to select sample stars which seem to fit a simple velocity-field model of the Galaxy, and to determine first the rotation of the reference frame.

It is known that K-M giants are an old and well-relaxed population of stars and are expected to have already reached a steady-state in the galactic potential. Such a state of a stellar system means that the system should exhibit only a simple plane-parallel galactic rotation described by the Oort constants $A=D_{12}^{+}$and $B=D_{21}^{-}$. In Paper I, we analyzed the proper motions of about $30000 \mathrm{~K}-\mathrm{M}$ giants chosen from the astrometric catalogue ACRS Part 1 (Corbin and Urban 1991) on the FK5 system. Starting from an initial trial of the correction $\Delta p \sim-0^{\prime \prime} .3 /$ cy suggested independently of the stellar kinematics by the VLBI and LLR observations (McCarthy and Luzum 1991, Williams et al. 1991), we have proven that the K-M giants are, indeed, in a steady state. Then, applying the plane-parallel galactic rotation model to these stars, we have determined a rational set of the corrections $\Delta p$ and $\Delta e+\Delta \lambda$ to the FK5 system together with the Oort constants and the solar motion (see Table 1). 
TABLE 1. Kinematic Parameters Derived from Proper Motions Given by ACRS Part 1

\begin{tabular}{|c|c|c|c|}
\hline $\begin{array}{l}\text { Kinematic } \\
\text { Parameters }\end{array}$ & $\begin{array}{c}\text { Least Squares } \\
\text { Method } \\
\text { K-M Giants } \\
|z| \leq 0.5 \mathrm{kpc}\end{array}$ & $\begin{array}{c}\text { Maximum Likelihood } \\
\text { Method } \\
\text { K-M Giants } \\
|z|<1.0 \mathrm{kpc}\end{array}$ & $\begin{array}{c}\text { Least Squares } \\
\text { Method } \\
\text { Young Stars } \\
0.5 \mathrm{kpc} \leq r \leq 3.0 \mathrm{kpc}\end{array}$ \\
\hline$\Delta p(" / c y)$ & $-0.267 \pm 0.028$ & $-0.214 \pm 0.022$ & -0.27 (given) \\
\hline$\Delta e+\Delta \lambda(" / \mathrm{cy})$ & $-0.116 \pm 0.026$ & $-0.075 \pm 0.037$ & -0.12 (given) \\
\hline$S_{1}(\mathrm{~km} / \mathrm{s})$ & $+13.6 \pm 0.3$ & $+13.4 \pm 0.31$ & $+8.7 \pm 0.8$ \\
\hline$S_{2}(\mathrm{~km} / \mathrm{s})$ & $+23.3 \pm 0.3$ & $+20.3 \pm 0.38$ & $+15.9 \pm 0.8$ \\
\hline$S_{3}(\mathrm{~km} / \mathrm{s})$ & $+11.9 \pm 0.3$ & $+12.2 \pm 0.22$ & $+9.1 \pm 0.7$ \\
\hline$S_{\text {total }}(\mathrm{km} / \mathrm{s})$ & 29.5 & 26.7 & 20.3 \\
\hline$A=D_{12}^{+}("$ /cy $)$ & $+0.263 \pm 0.012$ & $+0.243 \pm 0.011$ & $+0.285 \pm 0.019$ \\
\hline$B=D_{21}^{-}(" /$ cy $)$ & $-0.176 \pm 0.010$ & $-0.193 \pm 0.010$ & $-0.260 \pm 0.015$ \\
\hline$V_{\theta}(\mathrm{km} / \mathrm{s})$ & $-177.1 \pm 6.2$ & $-175.7 \pm 6.0$ & $-219.9 \pm 9.8$ \\
\hline$D_{13}^{+}$ & 0 & 0 & $-0.059 \pm 0.011$ \\
\hline$D_{13}^{-}$ & 0 & 0 & $+0.059 \pm 0.011$ \\
\hline$D_{23}^{+}$ & 0 & 0 & $+0.039 \pm 0.010$ \\
\hline$D_{32}^{-}$ & 0 & 0 & $+0.039 \pm 0.010$ \\
\hline$D_{z z}\left(\mathrm{~km} / \mathrm{s} / \mathrm{kpc}^{2}\right)$ & - & $15.6 \pm 2.2$ & - \\
\hline$\sigma_{R}(\mathrm{~km} / \mathrm{s})$ & - & $+31.3 \pm 0.4$ & - \\
\hline$\sigma_{\theta}(\mathrm{km} / \mathrm{s})$ & - & $+25.2 \pm 0.5$ & - \\
\hline$\sigma_{z}(\mathrm{~km} / \mathrm{s})$ & - & $+21.2 \pm 0.5$ & - \\
\hline$\epsilon_{\mu \alpha}(" / c y)$ & - & $0.56 \pm 0.02$ & - \\
\hline$\epsilon_{\mu \delta}(" / \mathrm{cy})$ & - & $0.52 \pm 0.02$ & - \\
\hline $\begin{array}{l}\text { total number } \\
\text { adopted }\end{array}$ & 20292 & 22629 & 1892 \\
\hline
\end{tabular}

A reason why the FK5 system is still rotating should be discussed here: In his determination of the rotation left in the FK4 system, Fricke used 512 FK4/FK4 Sup stars, which are biased to young ages (about $60 \%$ of these stars are O-B stars, Supergiants, and Bright Giants). Fricke adopted the plane-parallel galactic rotation model, so that the stars chosen are implicitly assumed to be in a steady-state. If the stars perform rotations other than the familiar rotation given by the Oort constant $\mathrm{B}$, the model attributes all the rotation components in the galactic plane to the precessional and equinoctial motions of the reference frame. However, since Fricke's sample of the 512 stars is biased to young ages, the sample is not guaranteed to be in a steady-state. Furthermore, the nearest distance limit of $70 \mathrm{pc}$ is still not sufficient to avoid localized velocity fields (cf. Gould-belt), and the motion of the more distant young stars is, on the other hand, liable to be 
disturbed by the galactic warp (Miyamoto et al. 1993).

So long as the analysis is based upon least squares, we miss the important information, the velocity dispersion of stars. In order to determine the dispersion as well and to examine how the previous result (Paper I) is modified by inclusion of dispersion, Tsujimoto and Miyamoto (1994) have examined again the local kinematic parameters and the rotation of the FK5 system by the Maximum Likelihood Method (Murray 1983), on the basis of the same sample of $\mathrm{K}-\mathrm{M}$ giants as before.

We have obtained a similar result (Table 1) to our previous one within the formal standard error. However, the present result gives smaller corrections to the FK5 system than before. The velocity dispersions $\left(\sigma_{R}, \sigma_{\theta}\right.$, $\sigma_{z}$ ) thus determined for the K-M giants agree with the generally accepted values. We also estimated the error of the proper motion $\left(\epsilon_{\mu \alpha}, \epsilon_{\mu \delta}\right)$. The estimation agrees quite well with the error cited in the ACRS Part 1. Moreover, in the present case, we tried to estimate the second derivative, $D_{z z}$ in Table 1, of the galactic rotation $V_{\theta}$ with respect to the height $z \mathrm{kpc}$ from the galactic plane. The result gives the relation $V_{\theta}(z)=V_{\theta}(0)-16 z^{2} \mathrm{~km} / \mathrm{s}$, which implies a shearing rotation of the K-M giants.

Having determined the rotation remaining in the FK5 system, we can go one step further. On the basis of the proper motion data given by the ACRS Part 1 for about 2000 O-B stars, Supergiants, and Bright Giants (referred to as young stars), we have examined the kinematic behavior of the HI galactic warp, whose optical counterpart is considered to be the young stars.

Applying the corrections given in Paper I to the proper motions, we can now determine all three components of the general galactic rotation of the young stars (cf. eq.(6)). The result for the young stars is given in the fourth column of Table 1 . The rotation of the young stars is indicated by $D_{21}^{-}, D_{13}^{-}$, and $D_{32}^{-}$. As described in Miyamoto et al. (1993), the young stars, as the optical counterpart of the HI warp, are now streaming around the galactic center in an inclined plane of the kinematic warp with the velocity -225 $\mathrm{km} / \mathrm{s}$, and simultaneously the plane is rotating around the galactic center - sun - anticenter line in the increasing sense of the present warp with an angular velocity of $4 \mathrm{~km} / \mathrm{s} / \mathrm{kpc}$.

\section{The Galactic Dark Halo}

On the basis of the radial velocities of HII regions/reflection nebulae, Blitz (1979) and Brand and Blitz (1993) have demonstrated that the galactic rotation curve is not declining in the region exterior to the solar circle. 
Moreover, Hartwick and Sargent (1978) have used the radial velocities of outlying globular clusters and dwarf spheroidals to show that the velocity dispersion of the galactic satellites remains nearly constant out to about $60 \mathrm{kpc}$ from the galactic center. These results are considered to provide strong evidence that our Galaxy has a massive dark halo. However, different analyses made by Miyamoto et al. (1980) and Lynden-Bell et al. (1983) for the radial velocity data of the galactic satellites have not always resulted in evidence for a massive dark halo, and Lynden-Bell et al. have described the current situation as "Slippery Evidence on the Galaxy's Invisible Heavy Halo".

Now, if we could indeed determine the spatial motion (especially the proper motions) of the Magellanic System, its galactic orbital motion would provide an important constraint for the mass distribution of the dark halo out to $50 \mathrm{kpc}$ from the galactic center. Furthermore, the determination of the absolute proper motions of the Magellanic Clouds (member stars) is of great importance for estimating the spatial motion and distance of MACHO's, which are considerd to be a physical constituent of the halo.

The theoretical orbital motion of the system (Murai and Fujimoto 1980, Lin and Lynden-Bell 1982) predicts that the LMC should have a heliocentric proper motion of about 2 mas/yr pointing to the direction of trailing the Magellanic Stream. Recently, Kroupa et al. (1994) and Jones et al. (1994) have detected the proper motions of the LMC, resulting in the total amount of $\mu=1-2 \mathrm{mas} / \mathrm{yr}$ pointing to the direction of trailing the Magellanic Stream. The results are fortunately compatible with the theoretical prediction.

Kroupa et al. (1994) used proper motions taken from a modern astrometric catalogue, the PPM Catalogue, for 35 stars which are proven by Sanduleak to be LMC members (hereafter, referred to as Sanduleak stars). The PPM catalogue is compiled on the FK5 system. However, as we have shown recently (Paper I), the FK5 system still needs modification as regards its corrections for the precession and equinoctial motion (the second column of Table 1). Moreover, the proper motion systems of different astrometric catalogues usually differ systematically. Therefore, a different astrometric catalogue with improved precessional and equinoctial motions gives a different result for LMC proper motions. We have recently examined the LMC proper motions (Miyamoto and Sôma 1994), based on the other modern catalogue, the ACRS Part 1 (Corbin and Urban 1991).

In order to separate the differences between the PPM and ACRS Part 1 catalogues into systematic and random parts, we use the catalogue comparison method developed by Brosche (1966), which has been later modified into a more practical form (Bien et al. 1978) and applied in the construction of the FK5. The catalogue difference, ACRS Part 1 - PPM, is composed 
TABLE 2. Proper Motions of the Large Magellanic Cloud

\begin{tabular}{lccc}
\hline Data Source & $\begin{array}{c}\text { Proper Motion } \\
\text { System }\end{array}$ & $\begin{array}{c}\mu \text {-total } \\
(" / \text { cy })\end{array}$ & $\begin{array}{c}\text { PA from NGP } \\
(\text { degree })\end{array}$ \\
\hline $\begin{array}{l}\text { Photographic Plates } \\
\text { (Jones } \text { et al. 1994) }\end{array}$ & galaxies & $0.12 \pm 0.04$ & 282 \\
$\begin{array}{l}\text { PPM } \\
\text { (Kroupa et al. 1994) }\end{array}$ & FK5 & $0.16 \pm 0.09^{*}$ & 306 \\
$\begin{array}{l}\text { PPM } \\
\text { (Miyamoto, Sôma 1994) }\end{array}$ & improved FK5 & $0.15 \pm 0.09$ & 287 \\
$\begin{array}{l}\text { ACRS } \\
\text { (Miyamoto, Sôma 1994) }\end{array}$ & improved FK5 & $0.09 \pm 0.09$ & 156 \\
$\begin{array}{l}\text { ACRS } \\
\text { (Miyamoto, Sôma 1994) }\end{array}$ & improved FK5 & $0.55 \pm 0.33$ & 190 \\
$\begin{array}{l}\text { ACRS 6 stars } \\
\text { (Miyamoto, Sôma 1994) }\end{array}$ & & & 122 \\
\hline
\end{tabular}

* Kroupa et al. gave 0.17. But, the proper motions of PPM No. 354939 in their Table 1 is erroneous.

of four types of differences in $\alpha, \delta, \mu_{\alpha}$, and $\mu_{\delta}$ for about 250000 common stars all over the celestial sphere. These differences are expanded into triple orthogonal series, respectively, of products of Fourier, Legendre, and Hermite polynomials, and are tested for statistical significance, term by term, according to Brosche (1966).

We have examined about 300 terms of the series and adopted only statistically significant terms for the systematic difference in $\alpha, \delta, \mu_{\alpha}$, and $\mu_{\delta}$ under the significant level of $0.1 \%$ in the F-test. Details of the comparison will appear elsewhere (Miyamoto and Sôma 1994). Figures 1 and 2 show the contours of the systematic difference of $\mu_{\alpha} \cos \delta$ and $\mu_{\delta}$, respectively, between ACRS Part 1 and PPM in the LMC region. The asterisks in the figures indicate the 35 Sanduleak stars. It is found that the systematic difference of the proper motions between ACRS Part 1 and PPM amounts to the value to be determined for the LMC proper motions.

Adding the systematic difference $\Delta \mu_{\alpha} \cos \delta$ and $\Delta \mu_{\delta}$ thus obtained to the proper motions given by the PPM for the Sanduleak stars, we have found the mean proper motions of the LMC on the ACRS system. Table 2 compares the LMC proper motions reported hitherto by various authors. In the table, the first column gives data sources and authors, and the second column the proper motion system used, where "galaxies" indicates the 


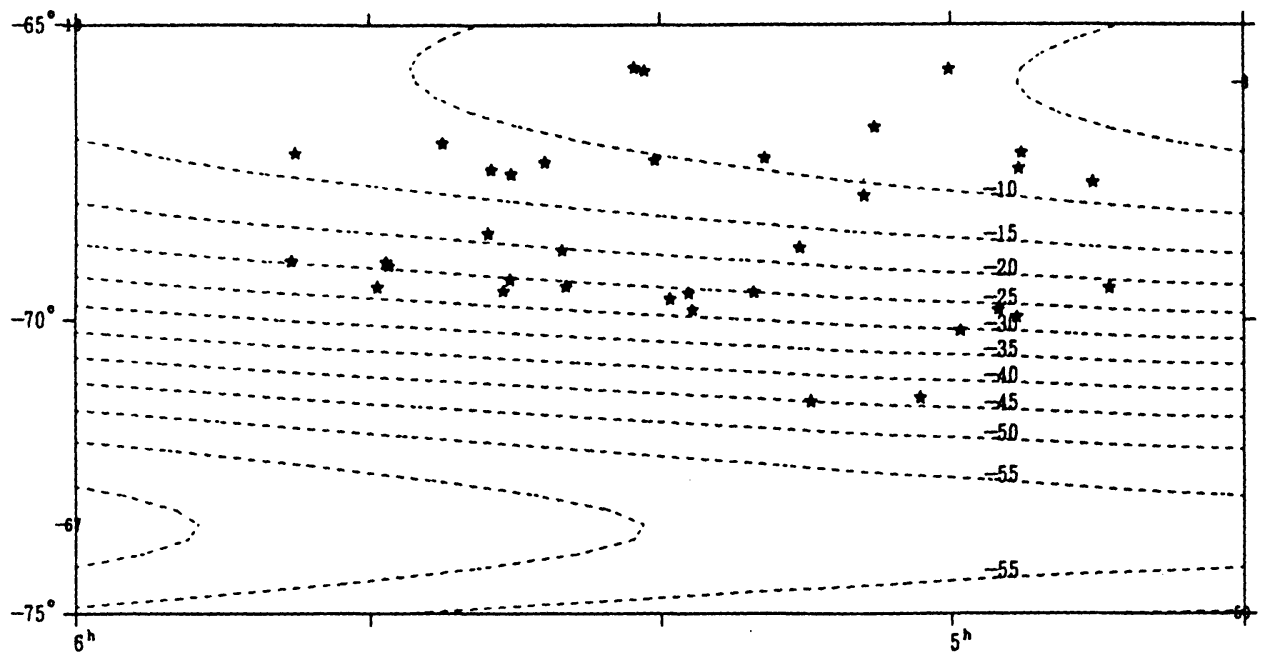

Figure 1. $\Delta \mu_{\alpha} \cos \alpha$ (ACRS-PPM): Tested are 293 orthogonal functions, of which 163 functions are selected. (unit: $0^{\prime \prime} .01 / \mathrm{cy}$ )

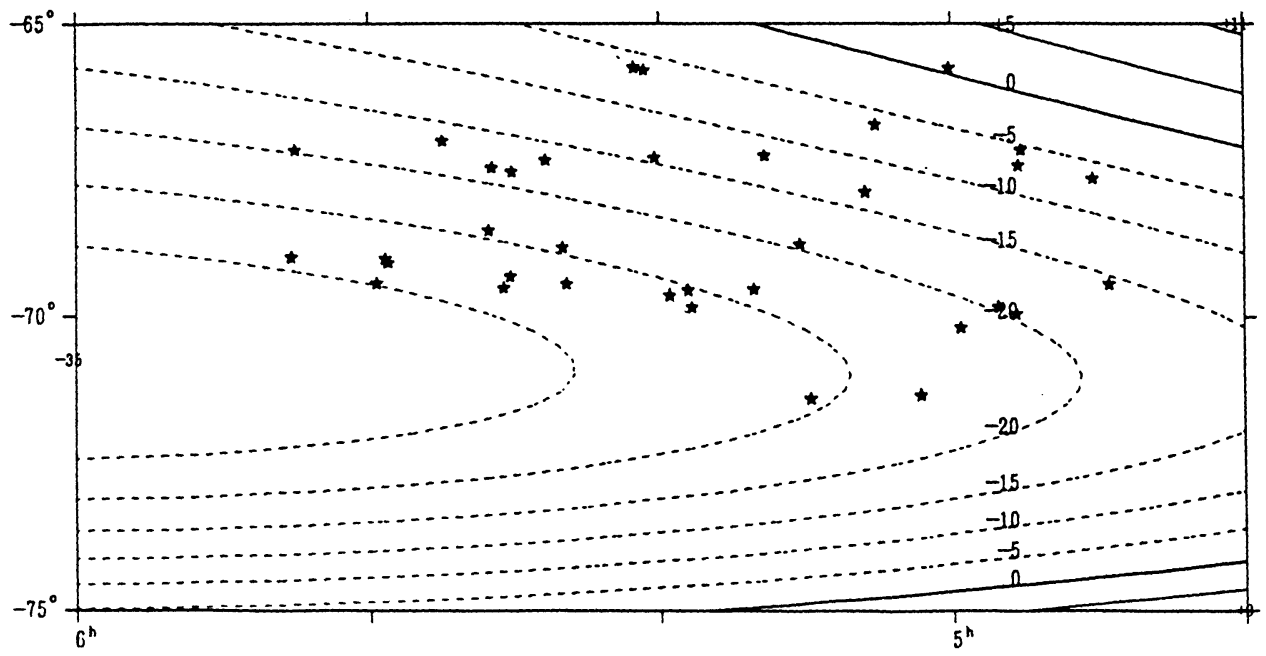

Figure 2. $\Delta \mu_{\delta}$ (ACRS-PPM): Tested are 293 orthogonal functions, of which 143 functions are selected. (unit: $0^{\prime \prime} .01 / \mathrm{cy}$ ) 
proper motions with reference to galaxies, and "improved FK5" means that the FK5 proper motion system was improved by introducing the corrections determined in Paper I.

Comparing the second and third lines in Table 2, we find that the corrections $\Delta p$ and $\Delta e+\Delta \lambda$ to the FK5 system do not damage the result given by Kroupa et al. (1994), but the corrections considerably affect the $\mu_{\alpha}$-component. The same is true for other cases. The fourth and fifth lines in Table 2 give the results based on the proper motion system of the ACRS Part 1. The remarkable point here is that the direction of the proper motion vector is almost reversed in comparison with the results given by the first to the third line. The last line gives, only for reference, the mean proper motions of 6 Sanduleak stars found in the ACRS Part1.

Though the present results are marginal in view of the standard error, the largest contrast with the previous results is that the ACRS proper motion system makes the LMC move toward the Magellanic Stream, contrary to the theoretical prediction. But, we remember that Lin and Lynden-Bell (1977) have once simulated the Magellanic Stream advancing ahead of the LMC. The LMC proper motions detected so far are not yet definitive.

\section{References}

Bien, R., Fricke, W. and Schwan, H. (1978) Veröff.Astron.Rechen-Institut Heidelberg, No. 29.

Blitz, L. (1979) ApJ, 231, L115.

Brand, J. and Blitz, L. (1993) A\&A, 275, 67.

Brosche, P. (1966) Veröff.Astron.Rechen-Institut Heidelberg, No. 17.

Corbin, T.E. and Urban, S.E. (1991) Astrographic Catalogue Reference Stars (ACRS), U.S. Naval Observatory.

Fricke, W. (1977) Veröff.Astron.Rechen-Institut Heidelberg, No. 28.

Fricke, W., Schwan, H. and Lederle, T. (1988) Veröff.Astron.Rechen-Institut Heidelberg, No. 32.

Hartwick, F.D.A. and Sargent, W.L.W. (1978) ApJ, 221, 512.

Jones, B.F., Klemola, A.R. and Lin, D.N.C. (1994) AJ, 107, 1333.

Kroupa, P., Röser, S. and Bastian, U. (1994) MNRAS, 266, 412.

Lieske, J.H., Lederle, T., Fricke, W. and Morando, B. (1977) A\&A, 58, 1.

Lin, D.N.C. and Lynden-Bell, D. (1977) MNRAS, 181, 59.

Lin, D.N.C. and Lynden-Bell, D. (1982) MNRAS, 198, 707.

Lynden-Bell, D., Cannon, R.D. and Godwin, P.J. (1983) MNRAS, 204, 87p.

McCarthy, D.D. and Luzum, B.J. (1991), AJ, 102, 1889.

Miyamoto, M., Satoh, C. and Ohashi, M. (1980) A\&A, 90, 215.

Miyamoto, M. and Sôma, M. (1993) AJ, 105, 691 (Paper I).

Miyamoto, M., Sôma, M. and Yoshizawa, M. (1993), AJ, 105, 2138.

Miyamoto, M. and Sôma, M. (1994) to be published.

Murai, T. and Fujimoto, M. (1980) PASJ, 32, 581.

Murray, C.A. (1983) Vectorial Astrometry, Adam Hilger Ltd, Bristol.

Trumpler, R.J. and Weaver, H.F. (1953) Statistical Astronomy, Univ. California Press.

Tsujimoto, T. and Miyamoto, M. (1994) to be published.

Williams, J.G., Newhall, X.X. and Dickey, J.O. (1991) A\&A, 241, L9. 